
\title{
R Reseracth Sulure \\ Should Spring Water Cysts of the Mediastinum Require Excisional Resection?
}

Soichiro Kiya ( $\nabla$ prichanprichan@gmail.com )

Shunan Memorial Hospital

Kembu Nakamoto

Shunan Memorial Hospital

Toshiyuki Fujii

Shunan Memorial Hospital

Eriko Sakka

Shunan Memorial Hospital

Yousuke Tsutsumi

Shunan Memorial Hospital

Kazuya Yoshida

Shunan Memorial Hospital

\section{Research Article}

Keywords: spring water cysts, mediastinal cysts, fenestration

Posted Date: February 18th, 2021

DOI: https://doi.org/10.21203/rs.3.rs-197768/v1

License: (c) (i) This work is licensed under a Creative Commons Attribution 4.0 International License.

Read Full License 
Title: Should Spring Water Cysts of the Mediastinum Require Excisional Resection?

Authors: Soichiro Kiya, MD", Kembu Nakamoto, MD, PhD", Toshiyuki Fujii, MD, PhD", Eriko Sakka, $\mathrm{MD}, \mathrm{PhD}^{2}$, Yousuke Tsutsumi, $\mathrm{MD}, \mathrm{PhD}^{2}$, Kazuya Yoshida, $\mathrm{MD}, \mathrm{PhD}$

Institutions and Affiliations: Division of General Thoracic Surgery"), Division of Anesthesiology ${ }^{2}$, Shunan Memorial Hospital, 1-10-1 Ikunoyaminami, Kudamatsu-shi, Yamaguchi, 744-0033 Japan

TEL $+8833-45-3330 \quad$ Fax $+8833-45-3331$

Classifications: Case report

Corresponding Author: Soichiro Kiya, Division of General Thoracic Surgery, Shunan Memorial Hospital, 1-10-1 Ikunoyaminami, Kudamatsu-shi, Yamaguchi, 744-0033 Japan E-mail: prichanprichan@gmail.com

Keywords: spring water cysts, mediastinal cysts, fenestration 


\section{Abstruct}

Complete surgical excision is the standard therapy for mediastinal cysts. Translucent cysts containing crystal-clear fluid are called "spring water cysts." We considered that these cysts can be treated adequately using fenestration with minimal bleeding and damage to existing structures. We experienced a case of mediastinal spring water cyst fenestrated under video-assisted thoracoscopy with a miniaturized endoscope (mini-VATS), instead of excisional resection. Fenestration of the non-neoplastic mediastinal cyst under mini-VATS might be a less invasive radical procedure and an alternative to complete resection. We discuss the indication for surgery and the surgical procedure for non-neoplastic mediastinal cyst with crystal-clear fluid.

\section{Introduction}

Mediastinal cysts account for 20-32\% of all mediastinal lesions [1], including thymic cysts, bronchogenic cysts, mature cystic teratoma, pericardial cysts, and esophageal duplication cysts. The symptomatic rate for these cysts is approximately 20\%. Asymptomatic mediastinal cysts are removed excisionally because of malignant transformation, cyst infection, progressive growth, or spontaneous rupture [2]. Complete surgical excision is the standard therapy for mediastinal cysts, with a postoperative complication rate of $6-12 \%[2,3]$

Translucent cysts containing crystal-clear fluid are called "spring water cysts." We experienced a case of mediastinal spring water cyst fenestrated under video-assisted thoracoscopy with a miniaturized endoscope (mini-VATS) [4] as an alternative to excisional resection. We discuss the indication for surgery and the surgical procedure for non-neoplastic mediastinal cyst with crystal-clear fluid.

\section{Case}

A 49-year-old woman presented with back pain. Chest computed tomography (CT) revealed a posterior mediastinal mass measuring $4.2 \times 1.8 \times 3.2 \mathrm{~cm}$ closed to the tenth thoracic vertebra (Fig. 1a), while plain chest X-ray was normal. Chest magnetic resonance imaging (MRI) demonstrated hypo-intensity on T1-weighted images and hyper-intensity on T2-weighted images (Fig. 1b). It was estimated that the posterior mediastinal mass did not contain a tumor component. The tumor was growing and symptomatic; 
therefore, surgery was indicated.

The patient underwent surgery under general anesthesia. The patient was placed in a lateral decubitus position and fixed with a fixing mattress (Magic Bed, Okada Medical Co. Ltd., Tokyo) on the operating table. An artificial pneumothorax for scope introduction was produced by needle thoracentesis under atmospheric pressure. Two ports were created at the seventh and eighth intercostal spaces; each skin incision was 2-5 mm long. A mini thoracoscope (2.9 mm IDEAL EYES, Stryker Co., Kalamazoo, MI) was used. The mediastinal semilucent cyst existed at the anterolateral portion of the tenth thoracic vertebra. The fluid in the cyst $(10 \mathrm{~mL})$ was absorbed using a 16G needle; rapid cytology of the fluid showed no malignancies. The fluid was watery and crystal-clear without cell particles. A coin-sized fenestration was created using a pair of scissors and electrocautery. There was a risk of hemorrhage at the anterior surface of the vertebral body when the whole cyst was dissected due to the exposure of blood vessels on the luminal surface of the cyst. Surgical cyst fenestration without excision of the cyst was performed under mini-VATS. At the end of the procedure, a double-lumen silicon catheter (18F Phicon Samp Catheter, Fuji Systems Co., Tokyo) was inserted into the thoracic cavity.

The thoracic catheter was extubated on postoperative day 1 , and the patient was discharged on postoperative day 4 . The postoperative course was uneventful. Histopathologic evaluation of the resected specimen revealed a mesothelial cyst (Fig. 2a, b). The patient experienced complete relief of symptoms. Fluid accumulation in the cyst was not observed on CT images 3 months postoperatively (Fig. 3).

\section{Discussion}

\section{Mediastinal spring water cysts}

The appearance of the thin-walled, translucent cysts containing crystal-clear fluid gave rise to the name "spring water cysts." Greenfield and colleagues introduced the new term into the medical literature when they encountered a cyst filled with "crystal-clear fluid of watery consistency" in 1943 [5]. Thin-walled solitary cysts with a low uniform density can be diagnosed as congenital thymic cysts according to their appearance on CT and MRI [6]. However, Margaret, et al. demonstrated a high proportion of agreement $(78 \%)$ between the results of fine-needle aspiration biopsy from the mediastinum and subsequent histologic diagnoses for a wide variety of mediastinal lesions [7]. Therefore, the diagnosis of spring-water cysts can be confirmed by intraoperative cytology. 
In this case, surgery was performed under general anesthesia as a complete resection with unilateral lung collapse was predicted. Fenestration may not require lung collapse during the procedure. Fenestration under mini-VATS is a relatively simple and less invasive procedure; local infiltration anesthesia or intercostal nerve block [8] may be applicable for tracheal intubation anesthesia. Moreover, mini-VATS is repeatedly applicable for the recurrence of non-neoplastic cysts because of its simplicity and lower invasiveness.

\section{Complete resection vs. fenestration}

Complete resection of the cyst has the risk of bleeding or injury to the adjacent normal structures. In this case, the cyst made contact with the vertebral column, and there was the risk of bleeding from the segmental artery and rupture of the vertebral foramen. Kozu et al. reported that all 108 primary mediastinal cysts were resected completely and were recurrence-free after a mean follow-up of $41 \pm 26$ months [3]. Moreover, Esme et al. reported that all 32 patients were asymptomatic and recurrence-free [2]. Spring water cyst could be categorized as a functional hydrocele. In the case of a functional hydrocele, we believe that even if fluid is produced, the thoracic pleura is capable of absorbing the fluid, and the cyst wall might not recur even if the wall is left in place. Fluid drainage through fenestration may prevent recurrent fluid collection.

\section{Conclusion}

Fenestration of non-neoplastic mediastinal cysts under mini-VATS might be a less invasive radical procedure compared to complete resection.

\section{Declarations}

Conflict of interest: The authors have no conflicts of interest to declare.

Ethics approval, Consent to participate, Consent for publication: The institutional review board of our hospital granted ethics approval for this study (\#IRB2602, MSH March 24,2015), and individual patient consent was obtained. 
1. Strollo DC, Rosado-de-Christenson ML, Jett JR. Primary mediastinal tumors: partII. Tumors of the middle and posterior mediastinum. Chest. 1997; 112: 1344-1357.

2. Hidir Esme, Sevval Eren, Murat Sezer, Okan Solak. Primary Mediastinal Cysts. Clinical Evaluation and Surgical Results of 32 Cases. Tex Heart Inst J. 2011; 38: 371-374.

3. Yoshiki Kozu, Kenji Suzuki, Shiaki Oh, Takeshi Matsunaga, Yukio Tsushima, Kazuya Takamochi. Single Institutional Experience with Primary Mediastinal Cysts: Clinicopathological Study of 108 Resected Cases. Ann Thorac Cardiovasc Surg. 2014; 20: 365-369.

4. Kembu Nakamoto, Masazumi Maeda, Taku Okamoto, Kohtaro Kameyama, Ayanori Sugita, Eiichi Hayashi. Preoperative diagnosis with video-assisted thoracoscopy with miniaturized endoscopes in general thoracic surgery: a preliminary study. Chest. 1998; 114: 1749-55.

5. Greenfield L, Steinberg L, Touroff ASW. Spring water cyst of the mediastinum. J Thorac Surg. 1943; 12: 495-502.

6. Yuji Matsumura, Muneo Minowa, Osamu Araki, Youko Karube, Syunsuke Eba, Yasutsugu Notsuda, et al. Appropriate surgical approach to cystic thymic lesions. Kyobu Geka. 2012; 65: 939-43.

7. Margaret W Assaad, Liron Pantanowitz, Christopher N Otis. Diagnostic accuracy of image-guided percutaneous fine needle aspiration biopsy of the mediastinum. Diagn Cytopathol. 2007; 35: 705-9.

8. Eriko Tanaka, Yousuke Tsutsumi, Kembu Nakamoto. A Comparison of the Perioperative Management Between ultrasound-Guided Thoracic Paravertebral Block and Local Anesthesia for Video-Assisted Thoracic Surgery with a Miniaturized Thoracoscope under Non-Intubation Anesthesia. BAOJ Surg. 2017; 3: 023.

\section{Figures}

Fig. 1

a: Chest computed tomography revealed a posterior mediastinal mass (arrow) measuring $4.2 \times 1.8 \times 3.2 \mathrm{~cm}$ close to the tenth thoracic vertebra.

b: Chest magnetic resonance imaging revealed hypo-intensity on T1-weighted images and hyper-intensity on T2-weighted images.

Fig. 2: A single layer of mesothelial cell-covered cystic lesions with thicker collagenous tissue just below the mesothelial cells was observed, accompanied by the presence of vitrification. No evidence of 
malignancy was found. $(\mathrm{a}: \times 200, \mathrm{~b}: \times 400)$

Fig. 3: Chest computed tomography did not show fluid accumulation in the cyst 3 months after surgery. 


\section{Figures}

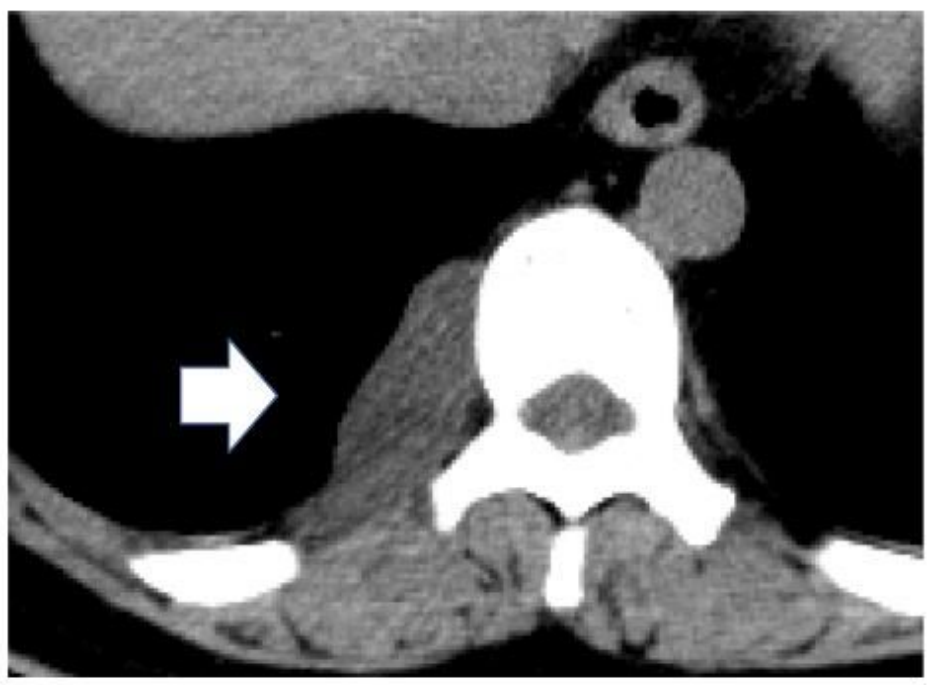

A

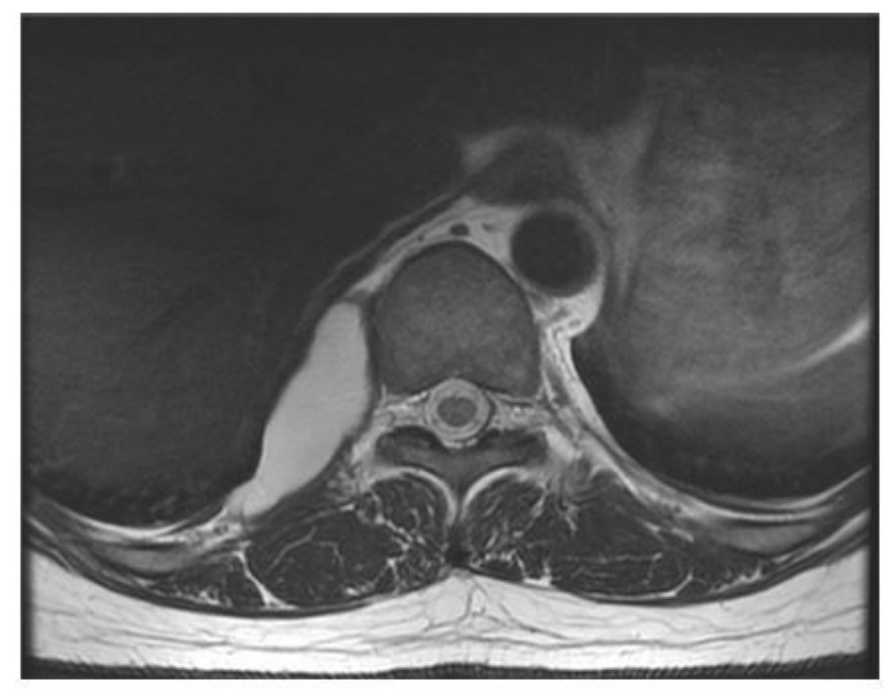

B

\section{Figure 1}

a: Chest computed tomography revealed a posterior mediastinal mass (arrow) measuring $4.2 \times 1.8 \times 3.2 \mathrm{~cm}$ close to the tenth thoracic vertebra. b: Chest magnetic resonance imaging revealed hypo-intensity on T1weighted images and hyper-intensity on T2-weighted images.

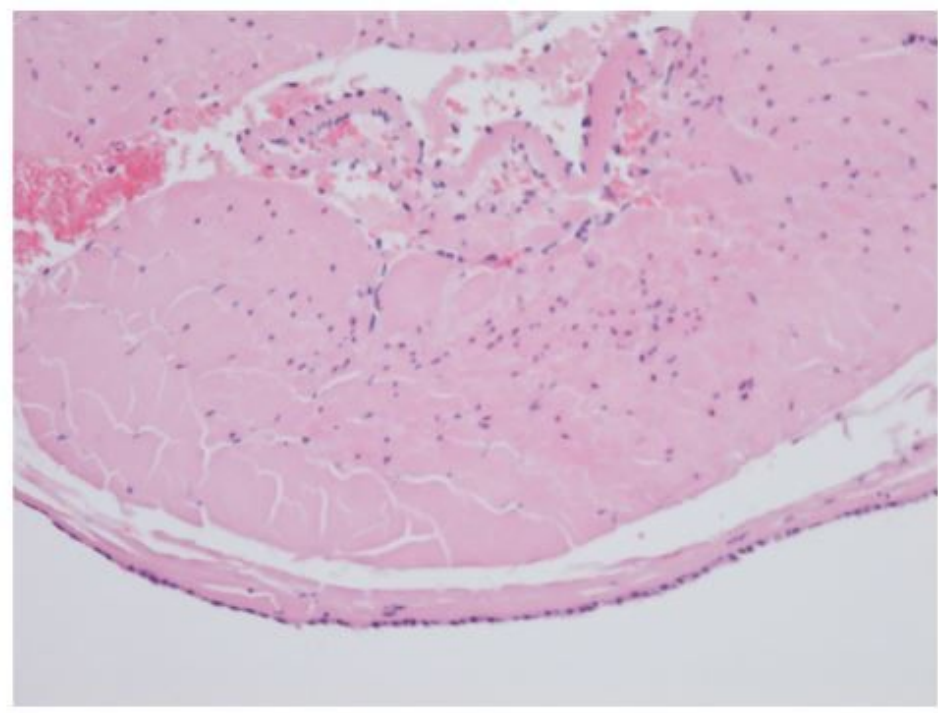

A

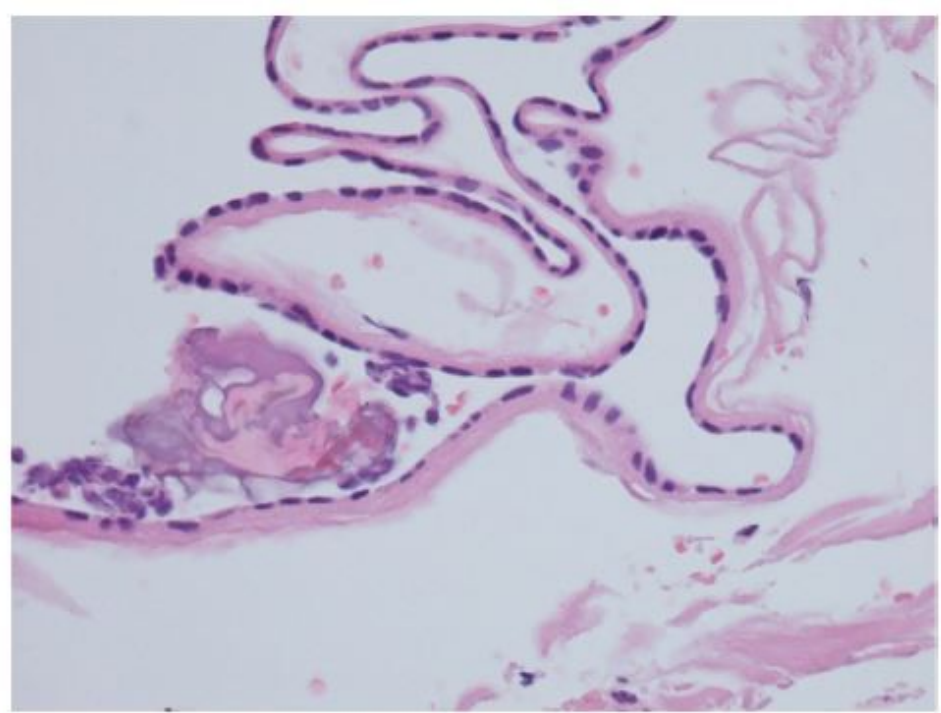

$\mathrm{B}$

\section{Figure 2}

A single layer of mesothelial cell-covered cystic lesions with thicker collagenous tissue just below the mesothelial cells was observed, accompanied by the presence of vitrification. No evidence of malignancy was found. $(a: \times 200, b: \times 400)$ 


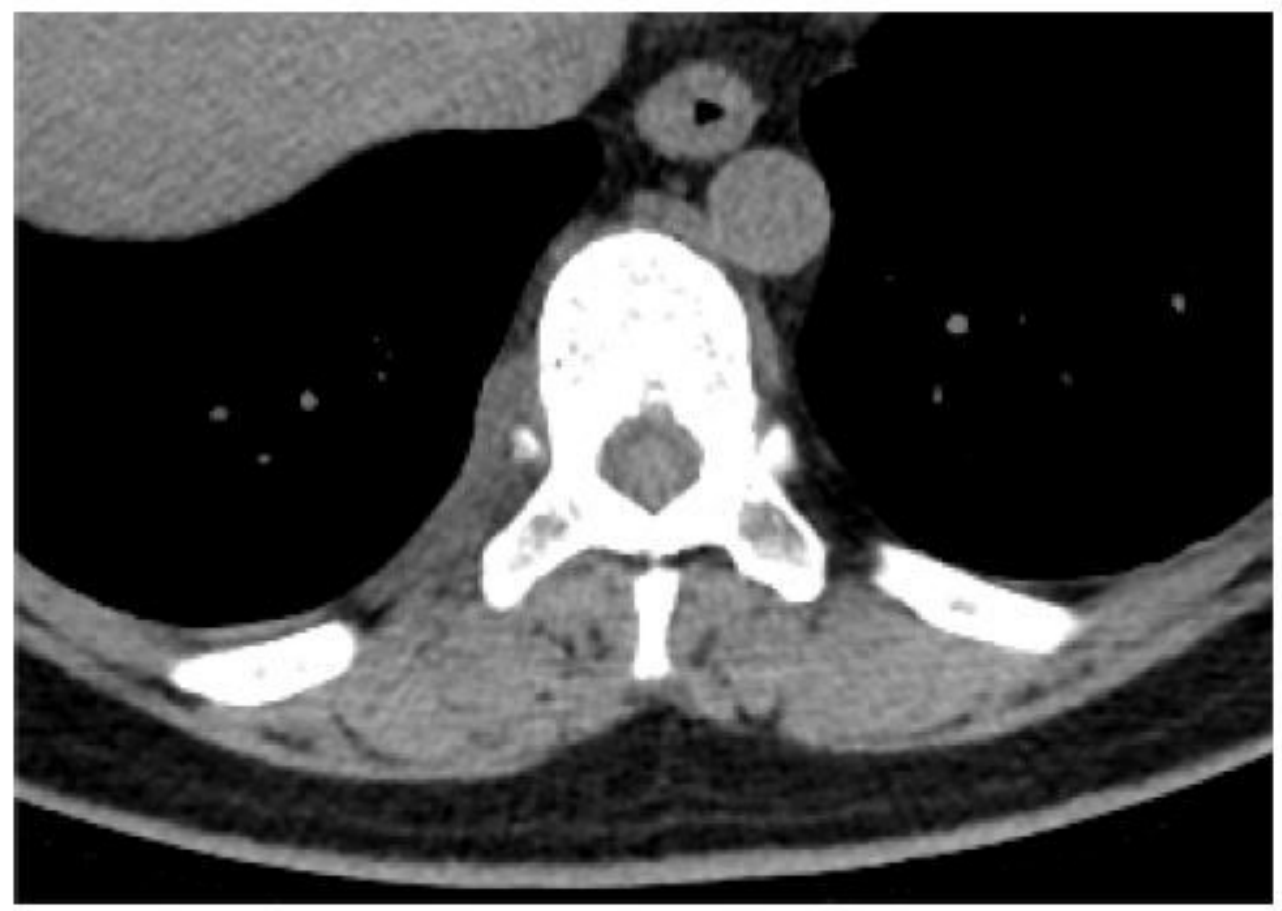

Figure 3

Chest computed tomography did not show fluid accumulation in the cyst 3 months after surgery. 